\title{
Lab-on-a-chip Raman sensors outperforming Raman microscopes
}

\author{
Ashim Dhakal ${ }^{1,2}$, Ali Raza ${ }^{1,2}$, Pieter Wuytens ${ }^{1,2,3}$, Frédéric Peyskens ${ }^{1,2}$, Andre Skirtach ${ }^{2,3}$, \\ Nicolas Le Thomas ${ }^{1,2}$ and Roel Baets ${ }^{1,2}$ \\ ${ }^{1}$ Photonics Research Group, INTEC Department-Gent University-imec, \\ ${ }^{2}$ Center for Nano- and Biophotonics, Ghent University, 9000 Gent, Belgium \\ ${ }^{3}$ Department of Molecular biotechnology, Ghent University, B-9000 Ghent (Belgium) \\ Authore-mail address: ashim.dhakal@ugent.be
}

\begin{abstract}
We demonstrate that the signal-to-noise ratio and signal collection efficiency in evanescent waveguide-based Raman spectroscopy exceeds that in Raman microscopes. We investigate the effect of silicon-nitride waveguide geometry to further improve the performance.
\end{abstract}

OCIS codes: (130.0130) Integrated Optics; (170.5660) Raman spectroscopy; (130.6010) Sensors.

\section{Introduction}

Single-mode nano-photonic waveguides have intrinsic assets to implement Raman spectroscopy in a lab-on-a-chip framework. They combine a large detection volume (Fig. 1) and field enhancement near the vicinity of a high-indexcontrast waveguides [1,2]. Further, smallest possible étendue of the collected signal in a single-mode waveguide allows smallest possible integrated spectrometers with an optimal spectral resolution. A high performance, low cost, and compact Raman sensor can thus be integrated on a chip using CMOS-compatible process steps. Here, we show that compared to the confocal microscopes, a $1 \mathrm{~cm}$ long silicon nitride $\left(\mathrm{Si}_{3} \mathrm{~N}_{4}\right)$ waveguide leads to more than three orders of magnitude higher signal. Such an enhancement of the signal contributes to a higher SNR for the waveguide systems compared to the dark-noise limited Raman microscopic systems. We also show that the use of the slotted waveguides increases the SNR and collection efficiency.
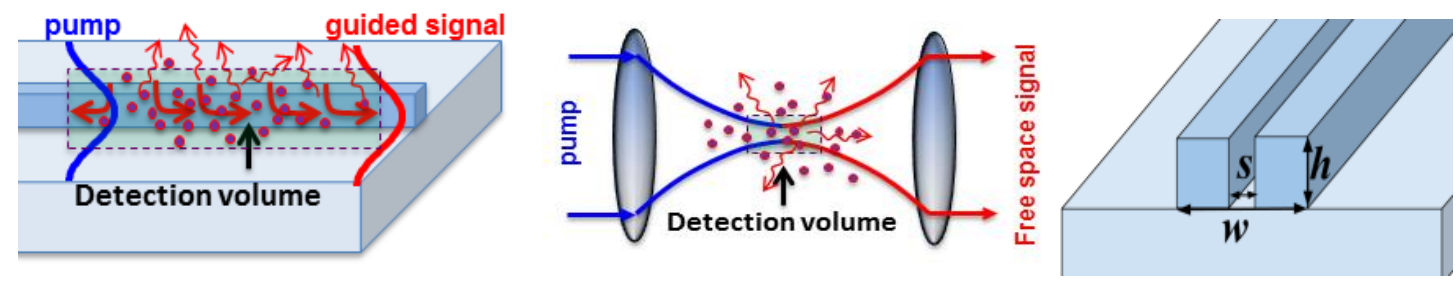

Figure 1: The detection volume for waveguide based evanescent Raman spectroscopy (Left) compared to that of the microscopes which is limited to the confocal volume (Middle). (Right) A generic slot waveguide. For strip waveguides, $s=0$.

\section{Experimental}

In our experiments, a laser pump at $785 \mathrm{~nm}$ excites the fundamental TE mode of the waveguide via an aspheric lens. The analyte, which is here pure isopropyl alcohol (IPA), is drop-casted on the waveguides and covers the relevant waveguide region. The Raman signal of the analyte is collected via the same waveguide. For strip waveguides a width $w$ of $700 \mathrm{~nm}$ is chosen while for slot waveguides, slot width $s=150 \mathrm{~nm}$, and total waveguide width $w=700$ is chosen. Both of the waveguides have the height $h=220 \mathrm{~nm}$. The waveguides are $1 \mathrm{~cm}$ in length and are coiled as spirals (typical size: $800 \mu \mathrm{m} \times 500 \mu \mathrm{m}$ ). To compare the on-chip approach with the conventional Raman microscope, we use a commercial confocal Raman microscope (WITec Alpha300R+). For the both cases, the same excitation laser wavelength and the same spectrometer (AvaSpec-ULS2048XL) are employed. An inverted configuration is used for the microscope to avoid any artefacts, which may result due to contact with the surface of the liquid analyte or due to evaporation of the analyte. IPA was contained in a Raman grade low background quartz receptacle with $0.17 \mathrm{~mm}$ bottom thickness. Several objectives corrected for 0.17 cover slip were are used for the measurement to optimally couple light in the $100 \mu \mathrm{m}$ diameter collection fiber that also acts as the pin-hole. Among 60x/0.8 NA, 20x/0.5NA and 10x/ 0.3 NA objectives, Nikon CFI Plan Fluor 10x/0.3 gave the highest signal and is used for the measurement of the spectra for comparison with the waveguide approach.

Figure 2 shows the Raman spectra of the IPA droplets collected using slotted waveguides, strip waveguides and the commercial microscope. The spectra are normalized by the respective values of the peak at $819 \mathrm{~cm}^{-1}$ which 
corresponds to the C-C-O vibration of IPA. Figure 2 also shows the plot of SNR versus collection efficiency, defined as the ratio of the Stokes power and the input pump power $\left(P_{S} / P_{i n}\right)[1,2]$ for various measured spectra for the $8191 / \mathrm{cm}$ peak of IPA. We verify that photon counts of the spectrometer-CCD follow Poisson statistics. Thus, we define SNR as:

$$
S N R \equiv \frac{C_{819}-C_{B}}{\sqrt{C_{819}}}
$$

where, $\mathrm{C}_{819}$ is the average number of photon counts of the $819 \mathrm{~cm}^{-1}$ IPA peak and $\mathrm{C}_{\mathrm{B}}$ is the average number of background photon counts near the bottom of the $819 \mathrm{~cm}^{-1}$ peak corresponding to the background from the waveguides. All the spectra are normalized for $10 \mathrm{~mW}$ input power and $10 \mathrm{~s}$ integration time. The effect of an estimated $8 \mathrm{~dB} /$ facet coupling losses in the measurement of the signal from the waveguides has also been taken into account to compare with the free-space approach.

In Fig. 2, we observe that the spectrum from the confocal microscopes contained only the dark-noise from the spectrometer and contained negligible background. This is anticipated because the signal was taken directly from IPA at the confocal volume and a low-background Raman grade quartz receptacle was used. In contrast, the spectrum collected from the waveguides is composed of the Raman signal from the analyte and the background originating from the $\mathrm{Si}_{3} \mathrm{~N}_{4}$ core of the waveguide [3]. Nevertheless, in comparison to the confocal microscopic systems, about 3 orders of magnitude higher signal is obtained from the waveguides resulting in a large improvement in the SNR. Note that compared to the strip waveguides, the slotted waveguides have lower background relative to the corresponding IPA peak. This can be explained by the strong enhancement of the field in the slotted region resulting in a stronger interaction with the analyte [2]. In contrast, for strip waveguides, the maximum intensity lies in the $\mathrm{Si}_{3} \mathrm{~N}_{4}$ core region thus collecting more background from it. Use of a slotted waveguide configuration also leads to about an eight-fold enhancement in the signal strength compared to the strip waveguide. Therefore, a significantly higher SNR can be observed for the slotted waveguides.
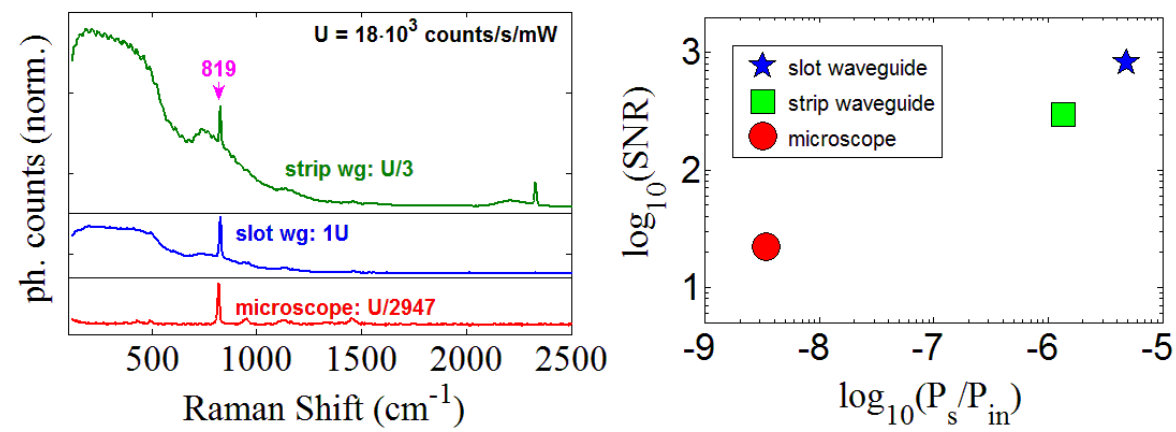

Figure 2: (Left) Raman spectra of IPA measured with the microscope (red, bottom), slot waveguide (blue, middle) and strip waveguide (green,top) and. The spectra are shifted vertically for clarity with their respective zeros shown by a black line, and normalized for the IPA peak at $819 \mathrm{~cm}^{-1}$ (shown with an arrow in magenta). (Right) The SNR vs collection efficiency for microscope (red circle), strip waveguide (green square) and slot waveguide (blue star).

\section{Conclusion}

In conclusion, because of the enhancement of the collected Raman signal in waveguide-based evanescent Raman spectroscopy, a higher SNR and collection efficiency are obtained in comparison to the confocal microscope despite the background noise from the waveguide core. The SNR can further be lowered by appropriate waveguide designs such as the slotted waveguide that enhances the interacting field intensity in the analyte region.

\section{References}

[1] A. Dhakal, et.al, "Evanescent excitation and collection of spontaneous Raman spectra using silicon nitride nanophotonic waveguides", Optics Letters, 39(13), p.4025-4028 (2014)

[2] A. Dhakal, et.al, "Efficiency of evanescent excitation and collection of spontaneous Raman scattering near high index contrast channel waveguides", Optics Express, 23(21), p.27391-27404 (2015)

[3] D. L. Smith, et. al, "Mechanism of SiNx Hy Deposition from $\mathrm{NH}_{3}-\mathrm{SiH}_{4}$ Plasma”, J. Electrochemical Society, 137(2), 614-623 (1990). 